\title{
Vasodilator stress and left ventricular asynchrony
}

\author{
Saurabh Malhotra, MD, MPH, FASNC, ${ }^{\text {a,b }}$ and John M. Canty Jr., MD ${ }^{a, b}$ \\ ${ }^{a}$ Departments of Medicine, Biomedical Engineering and Physiology \& Biophysics, The VA WNY \\ Health Care System and the Clinical and Translational Research Center at the University at Buffalo, \\ Buffalo \\ ${ }^{\mathrm{b}}$ Division of Cardiovascular Medicine, Clinical and Translational Research Center, University at \\ Buffalo, Buffalo, NY
}

Received Sep 9, 2015; accepted Sep 9, 2015

doi: 10.1007/s12350-015-0292-3

\section{See related article, pp. 43-52}

Left ventricular asynchrony (LVAS) or dyssynchrony refers to abnormal myocardial activation during a cardiac cycle resulting in inhomogeneous left ventricular contraction and reduced left ventricular function. LVAS is common among patients with heart failure (HF) and a reduced left ventricular ejection fraction (LVEF). Its prevalence in HF populations has been reported to be greater than $70 \%$, with a much higher prevalence among those with left bundle branch block (LBBB). ${ }^{1}$ Studies of LVAS in HF populations have demonstrated its ability to predict HF outcomes and arrhythmic events. ${ }^{2-4}$

Gated radionuclide myocardial perfusion imaging is an established technique for the assessment of LVAS using the phase analysis technique of gated myocardial perfusion images. This essentially assesses the dispersion in the timing of myocardial contraction in individual segments during a cardiac cycle. Central to the assessment of LVAS by phase analysis is the relatively linear relationship between myocardial thickening and myocardial count density in all myocardial segments (i.e., brighter myocardium in systole). ${ }^{5}$ Thus, the time-activity curve of a myocardial segment is essentially its temporal thickening curve. However, the temporal resolution of

\footnotetext{
Reprint requests: John M. Canty Jr., MD, Division of Cardiovascular Medicine, Clinical and Translational Research Center, University at Buffalo, 875 Ellicott Street, Suite 7030, Buffalo, NY 14203; canty@buffalo.edu

J Nucl Cardiol 2017;24:53-6.

$1071-3581 / \$ 34.00$

Copyright (C) 2015 American Society of Nuclear Cardiology.
}

gated radionuclide imaging is inherently poor due to the usual 8- or 16-bin gating. This can be improved by Fourier transformation of the time-activity data to generate a continuous thickening curve that delineates the timing of segmental myocardial contraction. ${ }^{6,7}$ This thickening curve is generated for over 600 myocardial voxels during a standard myocardial perfusion acquisition. With this approach, the initiation of contraction can be determined and compared among segments and a phase distribution (histogram) is generated. There are two widely used and validated indices of LVAS from phase analysis. One is the phase histogram band width (HBW) which is the range (in degrees) during which $95 \%$ of the myocardial voxels initiate contraction. The other is the phase standard deviation (SD) which is the standard deviation (in degrees) of the timing of contraction from all the myocardial voxels. ${ }^{8}$ Most of the data on LVAS comes from gated SPECT but a similar approach has been applied to gated PET perfusion imaging and the LVAS cut-offs for a normal population have been previously reported. ${ }^{9}$ Quantitative estimates of myocardial blood flow (MBF) for both rest and pharmacologicalstress images, and coronary flow reserve (CFR) are added advantages available with PET phase analysis.

Most studies of LVAS have focused on HF populations where it has shown to predict outcomes, ${ }^{4}$ malignant arrhythmias ${ }^{2,3}$ and the potential to guide resynchronization therapy. ${ }^{4,10}$ A few studies have evaluated LVAS in relation to ischemia on stress perfusion imaging. In an early SPECT study, no significant differences in LVAS were noted between gated rest and exercise stress studies despite the presence of significant ischemia in $50 \%$ of the study population. This negative result likely reflects normalization of any transient wall motion abnormalities since the stress images are acquired 45-60 minutes after tracer injection during 
exercise. ${ }^{11}$ In a subsequent larger study of patients with ischemic cardiomyopathy ( $\mathrm{LVEF}<35 \%$ ), changes in LVAS between rest and vasodilator stress were identified using Rubidium-82 (Rb-82) PET. $^{12}$ Normally perfused ventricles decreased LVAS whereas the development of ischemia resulted in increased LVAS. Interestingly, the change in LVAS during vasodilator stress was inversely related to the improvement in LVEF and was associated with all-cause mortality. The increased LVAS with pharmacological vasodilation was attributed to ischemia. Unlike exercise stress which results in ischemia due to an imbalance between myocardial oxygen delivery and demand, ischemia during pharmacological vasodilation is relatively uncommon. It sometimes develops due to a transmural steal phenomenon with relative subendocardial ischemia developing due to subepicardial vasodilation distal to a critical stenosis. ${ }^{13}$ It may also occur secondary to a decrease in diastolic perfusion pressure due to peripheral vasodilation along with tachycardia and increased contractility from reflex sympathetic activation. All of these factors could increase LVAS during vasodilator stress. On the other hand, improvement in LVAS with vasodilator stress is poorly explained. Previous studies of PET evaluating LVAS in patients without perfusion abnormalities have all reported an improvement in LVAS after vasodilator stress, regardless of baseline LVEF. $^{9,14}$ This may reflect altered LV loading conditions as well as enhanced myocardial contractility from reflex sympathetic activation or increased coronary flow (i.e., the Gregg Phenomenon). ${ }^{15,16}$ Thus, there are multiple ways that coronary arteriolar vasodilation can modulate measurements of LVAS.

In this issue of the journal, Van Tosh and colleagues evaluated the relation among indices of myocardial perfusion and LVAS in patients with largely preserved global $\mathrm{LV}$ function using phase analysis of gated rest/stress $\mathrm{Rb}$ 82 PET data. ${ }^{17}$ The authors retrospectively analyzed data from 195 patients referred for the evaluation of known or suspected coronary artery disease (CAD). Rest and stress myocardial blood flow (MBF) was calculated from first pass data and global MBF was defined as the average of the $\mathrm{MBF}$ values for the entire left ventricle. MBF heterogeneity was calculated as the ratio of the standard deviation of segmental MBF values to the average MBF value from 14 of the standard LV segments. Rest and stress HBW was used to define the presence or absence of LVAS, based on prior published cut-offs from a normal population. ${ }^{9}$ Greater than half the subjects had known $\mathrm{CAD}$ and at least one-third had angina, which likely prompted the referral for myocardial perfusion imaging (MPI). Interestingly, 54\% of the study population had LVAS on at least one of the scans despite global EF being fairly preserved. Based on the rest and stress phase analysis, the authors identified 4 patterns-no LVAS $(\mathrm{N}=89,46 \%)$, LVAS at rest which normalized with stress $(\mathrm{N}=13,7 \%)$, LVAS that developed during stress $(\mathrm{N}=42,21 \%)$ and LVAS that was present at rest and stress $(\mathrm{N}=51,26 \%)$. Patients without any LVAS had a higher rest $\mathrm{MBF}$ and lower rest coronary vascular resistance (CVR) when compared to the other subgroups. A summary of the major results comparing LVAS and myocardial perfusion are summarized in Figure 1.

Most patients who had LVAS at rest $(\mathrm{N}=64)$, continued to remain asynchronous with vasodilator stress (81\%). A small number that had LVAS which decreased with vasodilator stress $(\mathrm{N}=13)$ had significantly higher values of CFR, stress MBF and lower CVR as compared to those who continued to have LVAS during stress. Patients having LVAS that did not improve during vasodilator-stress had a significantly lower MBF and CFR. In analyzing the entire cohort, rest and stress MBF had a statistically significant, but modest correlation with rest and stress HBW. An LVEF $<55 \%$ during stress was most predictive of increased LVAS on receiver operating curve $(\mathrm{ROC})$ analysis $(\mathrm{ROC}$ area $=90 \%)$. An interesting finding of this study was a strong relationship between a

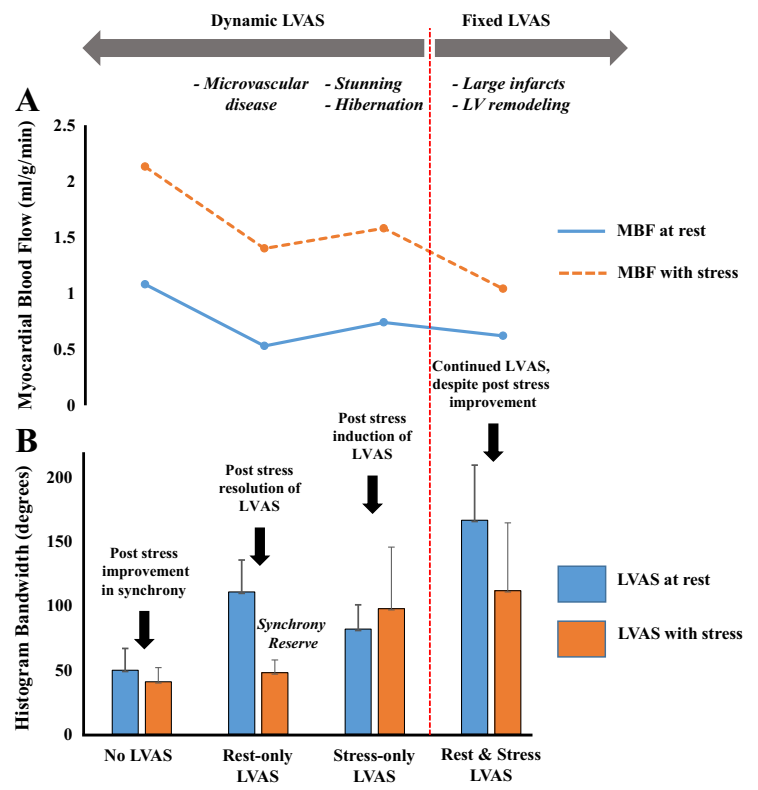

Figure 1. Relationship between left ventricular asynchrony (LVAS) and myocardial blood flow (MBF). Representation of the continuum of LVAS and its relation to MBF in a population with coronary artery disease. Values of HBW and MBF are taken from Van Tosh et $\mathrm{al}^{17}$. The population is stratified by the presence of LVAS at rest or with stress. MBF (A) and histogram bandwidth (HBW) (B) data are graphed across LVAS strata. 'Synchrony reserve' applies to reduction in LVAS after stress among patients with baseline LVAS, that could result in complete resolution of LVAS in a subset of the population. Abnormally high BHW characterizes patients with fixed LVAS, despite improvement in HBW after stress. 
stress-induced reduction in $\mathrm{MBF}$ heterogeneity with improvement in LVAS (ROC area $=84 \%$ ). The latter finding raises the possibility that in some circumstances, there is "synchrony reserve".

The manuscript highlights the reduction in vasodilated perfusion heterogeneity as a significant predictor of reduction in LVAS. Although the concept of "synchrony reserve", is provocative and biologically plausible, the results of this retrospective analysis are derived from a highly selected population with a high prevalence of CAD that is subject to referral bias and makes the observation less reliable. Furthermore, the prevalence of LBBB as well as a longer QRS durations (both important determinants of LVAS), are somewhat higher than observed in a cross sectional population of patients with chronic coronary artery disease without severely reduced LV function and potentially confounds the broad extrapolation of these findings. Another significant limitation is the fact that the perfusion measurements are global rather than regional and they employ a vasodilator stress where a supply demand imbalance and subendocardial ischemia is infrequently produced. Establishing a relationship between regional (segmental) changes in LVAS and segmental changes in MBF with exercise stress, where demand-induced ischemia develops and regional wall thickening diminishes, would provide stronger evidence for the relationship between $\mathrm{MBF}$ heterogeneity and heterogeneity in LVAS. Such an analysis would also clarify whether rest-only LVAS in the setting of low resting $\mathrm{MBF}$ is due to microvascular dysfunction and abnormal coronary autoregulatory responses at rest. In the absence of these regional evaluations, the relationship between LVAS and global MBF are simply correlations. Finally, an analysis of the independent impact of LVAS as well as perfusion heterogeneity on survival would have provided insight into its prognostic role in a cohort without severely reduced LVEF but unfortunately was not reported. Despite these limitations, this study further reinforces the complex nature of LVAS following vasodilator stress in patients with CAD.

An improvement in LVEF with vasodilator stress (EF reserve) has previously been reported from PET MPI but the mechanism of such a change has not been established. ${ }^{18}$ The present study supports the notion that an improvement in LVEF is likely preceded by a reduction in LVAS or 'synchrony reserve'. Studies have shown EF reserve to be inversely related to the severity of ischemia or infarction. ${ }^{12,19}$ Contrary to the systematic evaluation LVEF reserve and its prognostic value, ${ }^{18}$ the presence of a synchrony reserve is hypothetical and its clinical value is unknown. A prospective and more structured evaluation of LVAS in patients with CAD will be needed to determine whether it has a diagnostic and prognostic role in patient management when global LV function is relatively preserved. While speculative, the high prevalence of LVAS in this cohort with an LVEF $>35 \%$ raises the possibility that improvements in LVAS with CRT may be possible in a subpopulation with CAD and relatively preserved function. This, however, needs to be established in randomized prospective studies.

In summary, the study by Van Tosh and colleagues highlights the prevalence of LVAS in patients with relatively preserved LVEF as well as the potential impact of perfusion heterogeneity during vasodilator stress. Since LVAS assessed by radionuclide imaging is a predictor of adverse outcomes in HF populations, advancing our understanding of, its diagnostic, prognostic and therapeutic role in a population with hemodynamically significant CAD and preserved LV function could provide important information which may compliment the known risk of perfusion deficit size on cardiovascular outcomes. Future prospective studies to evaluate this should be encouraged.

\section{Acknowledgments}

Supported by the National Heart Lung and Blood Institute (HL-055324, HL-061610), the Department of Veterans Affairs and the Albert and Elizabeth Rekate Fund in Cardiovascular Medicine.

\section{References}

1. Malhotra S, Pasupula D, Khanna M, Soman P. Is left bundle branch block related to the mechanism of left ventricular dyssynchrony? J Am Coll Cardiol 2014;63:A1121.

2. Aljaroudi WA, Hage FG, Hermann D, Doppalapudi H, Venkataraman R, Heo J, et al. Relation of left-ventricular dyssynchrony by phase analysis of gated SPECT images and cardiovascular events in patients with implantable cardiac defibrillators. J Nucl Cardiol 2010;17:398-404.

3. Malhotra S, Pasupula D, Sharma R, Saba S, Soman P. Left ventricular dyssynchrony predicts ventricular tachyarrhythmia inpatients with severely reduced ejection fraction. J Nucl Cardiol 2013;20:657-97.

4. Friehling M, Chen J, Saba S, Bazaz R, Schwartzman D, Adelstein $\mathrm{EC}$, et al. A prospective pilot study to evaluate the relationship between acute change in left ventricular synchrony after cardiac resynchronization therapy and patient outcome using a single-injection gated SPECT protocol. Circ Cardiovasc Imaging 2011;4: 532-9.

5. Galt JR, Garcia EV, Robbins WL. Effects of myocardial wall thickness on SPECT quantification. IEEE Trans Med Imaging 1990;9:144-50.

6. Soman P, Chen J. Left ventricular dyssynchrony assessment using myocardial single-photon emission CT. Semin Nucl Med 2014;44:314-9.

7. Chen J, Boogers MJ, Bax JJ, Soman P, Garcia EV. The use of nuclear imaging for cardiac resynchronization therapy. Curr Cardiol Rep 2010;12:185-91. 
8. Chen J, Garcia EV, Folks RD, Cooke CD, Faber TL, Tauxe EL, et al. Onset of left ventricular mechanical contraction as determined by phase analysis of ECG-gated myocardial perfusion SPECT imaging: Development of a diagnostic tool for assessment of cardiac mechanical dyssynchrony. J Nucl Cardiol 2005;12:68795.

9. Cooke CD, Esteves FP, Chen J, Garcia EV. Left ventricular mechanical synchrony from stress and rest 82Rb PET myocardial perfusion ECG-gated studies: Differentiating normal from LBBB patients. J Nucl Cardiol 2011;18:1076-85.

10. Zhou Y, Faber TL, Patel Z, Folks RD, Cheung AA, Garcia EV, et al. An automatic alignment tool to improve repeatability of left ventricular function and dyssynchrony parameters in serial gated myocardial perfusion SPECT studies. Nucl Med Commun 2013;34:124-9.

11. Aljaroudi W, Koneru J, Heo J, Iskandrian AE. Impact of ischemia on left ventricular dyssynchrony by phase analysis of gated single photon emission computed tomography myocardial perfusion imaging. J Nucl Cardiol 2011;18:36-42.

12. AlJaroudi W, Alraies MC, Menon V, Brunken RC, Cerqueira MD, Jaber WA. Predictors and incremental prognostic value of left ventricular mechanical dyssynchrony response during stress-gated positron emission tomography in patients with ischemic cardiomyopathy. J Nucl Cardiol 2012;19:958-69.

13. Canty JM Jr, Duncker DJGM. Coronary blood flow and myocardial ischemia. In: Mann DL, Zipes DP, Libby P, Bonow RO, editors. Braunwald's Heart Disease. 10th ed. Philadelphia, PA: Elsevier Publishing; 2014. p. 1029-56.

14. AlJaroudi W, Alraies MC, DiFilippo F, Brunken RC, Cerqueira MD, Jaber WA. Effect of stress testing on left ventricular mechanical synchrony by phase analysis of gated positron emission tomography in patients with normal myocardial perfusion. Eur J Nucl Med Mol Imaging 2012;39:665-72.

15. Gregg DE. Effect of coronary perfusion pressure or coronary flow on oxygen usage of the myocardium. Circ Res 1963;13:497-500.

16. Iwamoto T, Bai XJ, Downey HF. Coronary perfusion related changes in myocardial contractile force and systolic ventricular stiffness. Cardiovasc Res 1994;28:1331-6.

17. Van Tosh A, Votaw JR, Cooke CD, Reichek N, Palestro CJ, Nichols KJ. Relationships between left ventricular asynchrony and myocardial blood flow. J Nucl Cardiol 2015. doi:10.1007/ s12350-015-0270-9.

18. Dorbala S, Hachamovitch R, Curillova Z, Thomas D, Vangala D, Kwong RY, et al. Incremental prognostic value of gated $\mathrm{Rb}-82$ positron emission tomography myocardial perfusion imaging over clinical variables and rest LVEF. JACC Cardiovasc Imaging 2009;2:846-54.

19. Dorbala S, Vangala D, Sampson U, Limaye A, Kwong R, Di Carli MF. Value of vasodilator left ventricular ejection fraction reserve in evaluating the magnitude of myocardium at risk and the extent of angiographic coronary artery disease: A $82 \mathrm{Rb}$ PET/CT study. J Nucl Med 2007;48:349-58. 